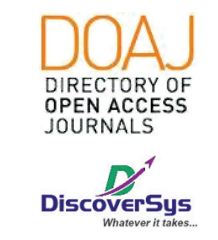

Published by DiscoverSys

\section{Ascites permagna caused by large mesenteric cyst in 2 years old male: A case report}

\author{
I Made Yullyantara Saputra, ${ }^{1 *}$ Putu Gde Karyana, ${ }^{1}$ Sanjaya Putra, ${ }^{1}$ \\ Metriani Nesa, ${ }^{1}$ Made Dharmajaya, ${ }^{2}$ Kadek Deddy Arianta ${ }^{2}$
}

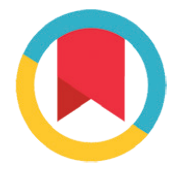

CrossMark

\title{
ABSTRACT
}

Background: Mesenteric cysts are documented as a rare entity in the pediatric population. They are considered as benign intraabdominal tumors with an unknown etiology and may occur anywhere in the mesentery of the gastrointestinal tract from the duodenum to the rectum. They are most commonly located in the mesentery of the ileum followed by localization in the sigmoid mesocolon. Approximately onethird of mesenteric cysts occur in children younger than 15 years of age and are slightly more common in males. The surgery is the common methods to remove the cyst.

Case presentation: A 2-year-old boy was hospitalized with a history of the bloated abdomen and dull achy pain on the left side of the abdomen since I year before admitted. Physical examination revealed distended of abdomen and ascites. Laboratory investigation showed normal limit; ultrasonography investigation showed cystic lesion and fluid collection in the cavum abdomen. It was confirmed with a CT scan. We diagnosed the patient with ascites permagna et causa suspected mesenteric cyst. After the diagnosis, a laparoscopic exploration and drainage ascites and total resection of the cyst were performed. Seven days after surgery, the patient was in good condition and discharged from the hospital - no distention in the abdomen.

Conclusion: The mesenteric cyst can be the cause of ascites and can be well treated with laparoscopic modalities; the outcome is excellent with no malignancy founded.
'Department of Child Health, Medical School of Udayana University, Sanglah Hospital, Bali, Indonesia

${ }^{2}$ Departement of Child Surgical, Medical School of Udayana University, Sanglah Hospital, Bali, Indonesia

\section{*Correspondence to:} I Made Yullyantara Saputra; Department of Child Health, Medical School of Udayana University, Sanglah Hospital, Bali, Indonesia; yullyantara.saputra@gmail.com

Received: 2019-03-16 Accepted: 2019-08-16 Published: 2019-08-01

Keywords: ascites, mesenteric cyst, laparoscopic surgery,

Cite This Article: Saputra, I.M.Y., Karyana, P.G., Putra, S., Nesa, M., Dharmajaya, M., Arianta, K.D. 2019. Ascites permagna caused by large mesenteric cyst in 2 years old male: A case report. Intisari Sains Medis 10(3): 537-542. D0I: 10.15562/ism.v10i3.447

\section{INTRODUCTION}

Mesenteric cysts are documented as a rare entity in the pediatric population. They are considered as benign intra-abdominal tumors with an unknown etiology. With an incidence of 1 case per 250,000 hospital admission, approximately 1:100,000 in adults and 1:20,000 in the pediatric population. ${ }^{1}$ They may occur anywhere in the mesentery of the gastrointestinal tract from the duodenum to the rectum, but they are most commonly located in the mesentery of the ileum followed by localization in the sigmoid mesocolon. Approximately one-third of mesenteric cysts occur in children younger than 15 years of age and are slightly more common in males. The size of mesenteric cysts may vary from $2 \mathrm{~cm}$ to $35 \mathrm{~cm}$. Italian anatomist Benevenni first described this entity performing an autopsy in an 8 -year-old boy in 1507, while Rokitansky published the first accurate description of a chylous mesenteric cyst in 1842 and Tillaux performed the first successful surgery for a cystic mass in the mesentery in $1880 .^{2}$

The exact etiology of mesenteric cyst has yet to be ascertained, but a failure of the lymph nodes to communicate with the lymphatic or venous systems or blockage of the lymphatics as a result of trauma, infection, and neoplasm are said to be contributing factors. The most popular theory, proposed by Gross, is a benign proliferation of ectopic lymphatics in the mesentery that lack communication with the remainder of the lymphatic system. ${ }^{1,2}$

A mesenteric cyst may occur in patients of any age. Approximately one-third of cases occur in children younger than 15 years. The cyst may present either as a non-specific abdominal feature, as an incidental finding, or as an acute abdomen. They are often asymptomatic and found incidentally while patients are undergoing workup or receiving treatment for other conditions, such as appendicitis, small bowel obstruction, or diverticulitis, although patients may present with lower abdominal pain and symptoms that are frequently associated with other abdominal conditions. The symptoms are variable and non-specific and include pain (82\%), nausea and vomiting (45\%), constipation (27\%), and diarrhea (6\%). An abdominal mass may be palpable in up to $61 \%$ of patients. ${ }^{5}$

The mesenteric cyst should be evaluated with complete history, clinical examination, blood investigations and radiological investigations (X-ray abdomen erect, ultrasound abdomen (USG) and computed tomography (CT) scan in selected cases) to reach a provisional diagnosis. The diagnosis is 
confirmed with laparotomy and has to be histologically confirmed. Secondary complications associated with mesenteric cysts include volvulus, spillage of infective fluid, herniation of bowel into an abdominal defect, and obstruction. The choice of treatment is complete excision to avoid recurrence and possible malignant transformation. Bowel resection may be necessary in cases which cysts are close to bowel structures or involve blood vessels that supply the bowel. Once removed, mesenteric cysts rarely recur, and patients have an excellent prognosis. Malignant cysts occur in less than 3\% of cases. Mesenteric cysts are most commonly single and multilocular, and the cystic fluid is generally serous when the cyst involves the distal small bowel or colonic mesentery; cystic fluid is usually chylous when the cyst is located in the proximal small bowel mesentery. Histopathological examination can confirm these observations

Complete surgical excision is the preferred treatment for mesenteric cysts and leads to excellent outcomes. Surgery can be performed laparoscopically. Bowel resection and anastomosis may be required along with removal of the mesenteric cysts. In children, resection and anastomosis may be needed in up to $50 \mathrm{e} 60 \%$ of cases. Rattan $\mathrm{KN}$ et al. reported 8 cases of mesenteric cysts, two of which presented with intestinal obstruction, both were treated by exploratory laparotomy, and complete excision of the cyst required bowel resection. If enucleation or resection is not possible, then partial excision with marsupialization of the remaining cyst into the abdominal cavity is recommended. The cyst lining could be sclerosed with electrocautery, tincture iodine, or OK432 after marsupialization to prevent recurrence. ${ }^{3,4,5}$

Primarily, no mortality is associated with mesenteric cysts in children; only one pediatric death has been reported since 1950. In a series from Egleston Children's Hospital in Atlanta, no major postoperative complications, recurrences, or deaths occurred. Based on those mentioned above, this case report aims to present mesenteric cyst, which can cause ascites permagna and could be well treated without complication.

\section{CASE-REPORT}

A 2-year-old boy, from Marga, Tabanan, Bali admitted with a history of the bloated abdomen and dull pain on the left side of abdomen since one year before admission. The pain mainly occurs after meals. A lump was noticed on the left side of the abdomen by a parent since the baby was born and the size increasing gradually. There was no history of fever, vomiting, jaundice, black stool, bloody vomit, rectal bleeding, dysuria, haematuria, chronic cough, bloody cough, bony pains, seizures, or worm infestation.

$\mathrm{He}$ was born spontaneously and vigorously at gestational age 38 weeks. Birth weight was 2500 grams, $45 \mathrm{~cm}$ of length, and $37 \mathrm{~cm}$ of head circumferences. Mother's age was 29 years old when she had the first pregnancy. Antenatal care was performed by an obstetrician every 3 months, and antenatal ultrasound revealed within normal. There were no risk factors of infection during pregnancy and bad obstetric histories. There were no complaints during pregnancy and no histories with other diseases associated with pregnancy. The patient was the first child and there where no complaint of his siblings.

On physical examination, the patient was alert. Respiratory rate was $55 \mathrm{x} /$ minute with a regular heart rate of 100 times/minute. His axillary temperature was $36.5^{\circ} \mathrm{C}$. Examination of the head and face was normal shaped. There where no conjunctiva injection, anemia nor icteric sclera. The pupil light reflects normal. The ear, nose, and throat examination were in normal limit. It was no cyanotic lips. The chest was symmetrical both on rest and movement; breath sound was bronchovesicular without rales or wheezing. The first heart sound was single and often accentuated. The second heart sound was narrowly split, with an accentuated pulmonary component. It was no murmur. In the abdomen, from percussion, we found all dullness, a fluid thrill or wave demonstrable in this case. We didn't find any signs of chronic liver disease such as palmar erythema, spider naevi, jaundice. It was a mild epigastric distention with normal turgor. Bowel sound was normal. Liver and spleen were difficult to evaluate. The extremities were normal. The power, tonus, and reflex of the superior and inferior extremities were normal - there was no enlargement of lymph nodes in all body area. The rectal was found normal in this patient. Based on history taking and physical examination, we concluded the working diagnosis was ascites permagna et causa suspect malignancy process $\mathrm{dd}$ abdominal cyst.

The patient underwent several laboratory examinations. Complete blood count revealed hemoglobin $(\mathrm{Hb})$ level was $11.3 \mathrm{~g} / \mathrm{dL}$, hematocrit $(\mathrm{Ht}) 32.3 \%$, leukocytes $6.1 / \mathrm{mm}^{3}$,thrombocytes $340 / \mathrm{mm} 3$, ESR $8 \mathrm{~mm} /$ hours, and C-reactive protein was $0.19 \mathrm{mg} / \mathrm{L}$. The liver function was within normal limit, serum aspartate transaminase (AST) $39 \mathrm{U} / \mathrm{L}$, alanine aminotransferase (ALT) $13 \mathrm{U} / \mathrm{L}$, albumin $4.1 \mathrm{~g} / \mathrm{dl}$, and alkali phosphatase (ALP) $228 \mathrm{U} / \mathrm{L}$. Electrolyte examination showed low level of Natrium 134 $\mathrm{mmol} / \mathrm{L}$, Kalium $(\mathrm{K}) 4.6 \mathrm{mmol} / \mathrm{L}$, Chloride $(\mathrm{Cl})$ $98.4 \mathrm{mmol} / \mathrm{L}$, and Calcium (Ca) $9 \mathrm{mg} / \mathrm{dl}$. Kidney function test revealed BUN $11 \mathrm{mg} / \mathrm{dL}$ and serum creatinine $0,36 \mathrm{mg} / \mathrm{dL}$, with glomerular filtration 

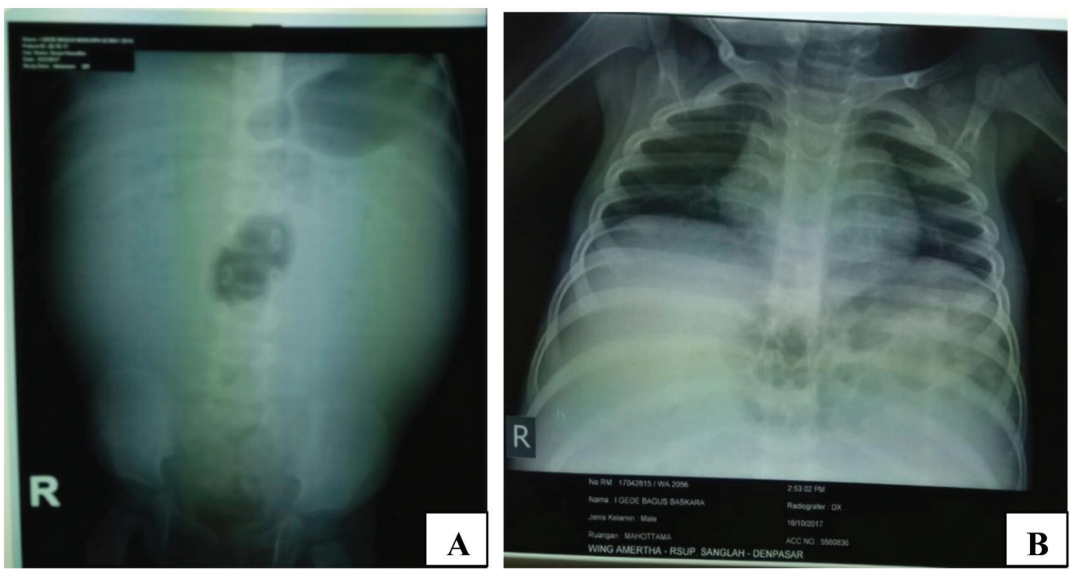

Figure 1 (A) abdominal radiography showed ground glasses appearance and (B) a thoracal X-ray showed normal lungs and heart
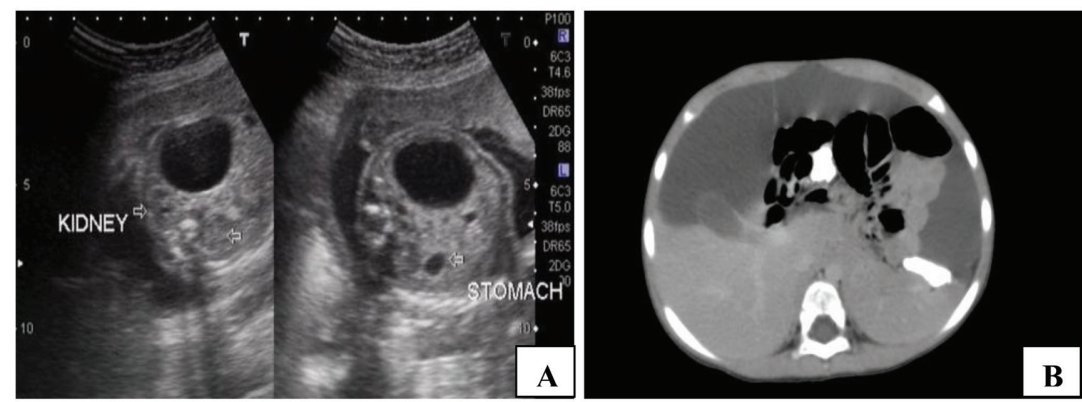

Figure 2 (A) Fluid collection with internal echo inside a cyst suggested a choric ascites and (B) Ascites permagna with the intraabdominal cyst
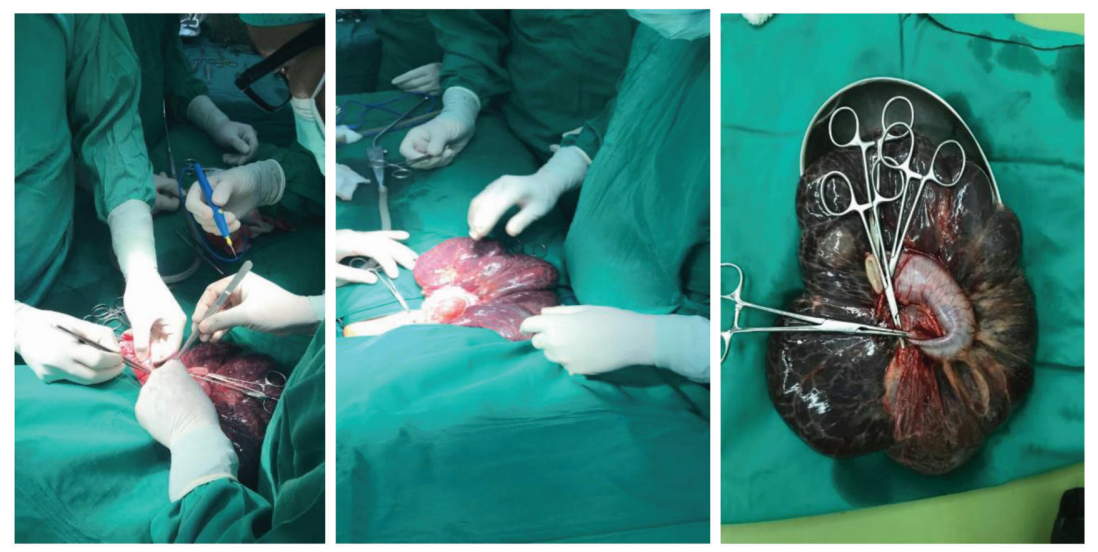

Figure 3 A mesenteric cyst found in the sigmoid colon

rate of GFR 90. Coagulation profile showed international normalized ratio (INR) of 0.97 with partial thromboplastin time (PTT) 12.3 (control 11.3) and increased level of activated partial thromboplastin time (APTT) 27 (control 45.0). Abdominal radiography revealed "ground glass appearance" in the cavum abdomen (Figure 1). Thoracal radiography showed cor and pulmo within normal limit (Figure 1). Abdominal ultrasound showed a fluid collection with internal echo inside a cyst suggested a choric ascites (dd mesenteric cyst), suggested to do MS CT-scan for further diagnosed (Figure 2).

Abdominal CT-Scan showed fluid accumulation in the abdominal cavity and a fluid-covered cyst mass in the abdominal cavity, suspect a mesenteric cyst. Organs inside the abdominal cavity (liver, bile, left and right kidneys, spleen, bladder, and prostate within normal limit) were shown in Figure 2. The conclusion from CT Scan was ascites permagna likely caused by abdominal cavity cyst.

Based on clinically laboratory result, the patient was diagnosed with ascites permagna et causa suspect mesenteric cyst. This patient was given antibiotic ceftriaxone 700mg BID. Blood culture was taken before antibiotic administration and consulted to the pediatric surgeon for evaluation and treatment. The pediatric surgeon diagnosed was a mesenteric cyst and planned to do laparoscopic diagnostic and drainage ascites.

At the $5^{\text {th }}$ day after admission inward, he underwent laparoscopic diagnostic and drainage ascites. In the surgery room, drainage ascites was followed with laparotomy exploration. A transverse incision at supra umbilical and abdominal exploration was conducted. The patient underwent exploratory laparotomy, which revealed a big lobulated mesenteric cyst in the mesentery of sigmoid, extending in the mesentery of descending colon and send the specimen to pathology anatomy for diagnostic (gold standard) (Figure 3).

After surgery, the patient was admitted to the Pediatric Intensive Care Unit (PICU). The enteral feeding was postponed until the next 2 days. After surgery laboratory examination was performed, the leucocyte count revealed $11.7 \mathrm{~K} / \mathrm{uL}$, with absolute neutrophile count 15.1 (88.7\%), hemoglobin 11.45 $\mathrm{g} / \mathrm{dL}$, platelet count was $288 \mathrm{~K} / \mathrm{uL}$. Based on this condition, the addition of intravenous antibiotic therapy was metronidazole $150 \mathrm{mg}$ every 8 hours. Two site blood culture performed before antibiotic administration.

On the second day after surgery, he was in good condition, no fever, no bloating, and the bowel sound quite good. He tried to drink and well tolerated. And he was admitted to the ward. On the $7^{\text {th }}$ days' post-operation, he was discharged from hospital in good condition. The patient was scheduled for follow up in one week. Blood culture showed no growth, and the pathology anatomy result was the histomorphology suggestive for mesenteric cyst, and there was no malignancy cells.

\section{DISCUSSION}

Ascites is the pathological accumulation of free fluid in the peritoneal cavity. The fluid accumulates 
because of conditions directly involving the peritoneum (infection, malignancy), or due to other diseases remote from the peritoneum (i.e., liver disease, heart failure, hypoproteinemia). Typically there is no fluid in the peritoneal cavity, however in women, a small amount (almost $20 \mathrm{ml}$ ) or less than 1 ounce can sometimes (but not often) can be present depending on her menstrual cycle (Ascites-1). However, for the ascites confirmation, it is required that at least $1500 \mathrm{ml}$ of fluid should be present in the peritoneal cavity and also detectable by clinical examination but significantly more in an obese person. Ascites exists in three grades, grade 1 which is mild, only visible on ultrasound and CT scan, grade 2 which is determined with flank bulging and shifting dullness and grade 3 is directly visible, and is confirmed with the fluid wave/thrill test. Traditionally, ascites was divided into 2 types, transudate, and exudate type. This classification was based on the amount of protein found in the fluid. ${ }^{1}$ In this case, a two-year-old male patient complained bloated abdomen since one year before admission that worsens by days. There was no sign and symptoms of liver failure, heart defects, nor infection. This case was Grade $3^{\text {rd }}$ Ascites, because of positive thrill test and it could directly be seen.

Mesenteric cysts are rare intra-abdominal lesions arising with an incidence of 1/100,000 admissions in adults and 1/20,000 in pediatrics. Many authors consider mesenteric, omental, and retroperitoneal cysts as one group because they derive from the same embryological structures whereas others defined mesenteric cysts as cysts arising in or near the mesentery with no connection to retroperitoneal organs. Although a few mesenteric cysts are well identified, controversy still exists about the etiology and classification of most of these cysts, but a failure of the lymph nodes to communicate with the lymphatic or venous systems or blockage of the lymphatics as a result of trauma, infection, and neoplasm are said to be contributing factors. The most accepted theory, proposed by Gross, is a benign proliferation of ectopic lymphatics in the mesentery that lack communication with the remainder of the lymphatic system. The most widely recognized classification was proposed in 1950 by Beahrs et al., They divided mesenteric cysts into 4 groups based mainly on clinical and etiological features: (1) embryonic and developmental; (2) traumatic; (3) neoplastic, and (4) infectious. ${ }^{1,2}$ In this case, These cysts include embryonic in origin, because the patient's father said the patient's stomach was already large since birth. From pathology anatomy assessment, it was confirmed that the diagnosis of the mesenteric case revealed because we saw lymphoplasmacytic cell inflammation in the mucosa.
Clinical presentation of the mesenteric cyst usually children generally present with abdominal distention and few associated symptoms other than vague abdominal pain with or without a palpable mass. The mass may be huge, simulating ascites. The most common mode of acute presentation in children is that of a small-bowel obstruction, which may be associated with intestinal volvulus or infarction. Approximately $10 \%$ of patients with mesenteric cyst present with an acute abdominal emergency. These masses can be detected with prenatal ultrasonography and appear as a sonolucent mass. The prenatal differential diagnosis includes the following, dilated bowel (e.g., intestinal atresia), dilated stomach (e.g., pyloric atresia), gastrointestinal duplication, hydronephrosis, ovarian cyst, and cystic teratoma. In a series of 82 children who underwent surgery for various causes of intestinal volvulus, mesenteric cysts were the underlying etiology in $3.65 \%$ of cases. ${ }^{3,4}$

The symptoms are variable, specific, and include pain (82\%), nausea and vomiting (45\%), constipation (27\%), or diarrhea (6\%). An abdominal mass may be palpable in up to $61 \%$ of the patients. They can present either with nonspecific abdominal complaints or with acute abdominal pain. The clinical presentation also depends on its associated complications. However, many of these cases are asymptomatic and diagnosed incidentally. Abdominal pain is documented as the most frequent symptom, and an acute abdominal presentation revealing a bowel obstruction is often reported in infants. Rarely abdominal distension or a mass are found on physical examination. ${ }^{4}$ In this case, main complaint was abdominal bloating. From anamnesis, the abdominal enlargement was being worsened by the last 12 months. This symptom accompanied by uncomfortable feelings, usually after a meal. There were no nausea, vomiting, constipation, nor diarrhea. From the physical examination, it was suggested for ascites. Abdominal mass could not be examined due to ascites. It was no sign of acute abdominal. The abdominal enlargement was a result of intraabdominal fluid accumulation. The fluid came from lymphatic system production of cysts those did not have a drainage system; then it accumulated inside the abdominal cavity. Extension fluid accumulation further compressed other intraabdominal organs, that caused nausea, vomiting, abdominal distention, and other obstruction signs. We performed a journal search to know about the clinical presentation of the gastrointestinal cyst. We founded a journal with title, "Cysts of Gastrointestinal Origin in Children: Varied Presentation" whereas the conclusion of the journal is abdominal pain was the most common presenting symptom. 
In tertiary perinatal centers where a prenatal diagnosis has already been established, no further diagnostic workup is typically necessary. Prenatal diagnosis is usually performed in the second half of pregnancy. There are only a few cases in which the diagnosis was made at the end of the first trimester or during the beginning of the second trimester. Fetal intraabdominal cysts are rare entities, and differential diagnosis is difficult. The concept of abdominal cyst encompasses many cystic lesions developing from abdominal structures and representing distinct pathologies. In differential diagnosis ovarian cysts, enteric duplication cysts, mesenteric cysts, meconium pseudocysts, and choledochal cysts should be considered apart from cysts with the renal origin. Adrenal cysts, splenic cysts, hydrocolpos, urachal cysts, and chylous ascites are seen less frequently. First line diagnostic tool is Ultrasonography (USG); however, more advanced imaging techniques such as magnetic resonance imaging (MRI) may prove useful in the management process. Due to more widespread usage of USG in the antenatal follow-up period, the incidence of ovarian cysts increased. Addition of MRI as a diagnostic tool significantly improved diagnosis and management. Monitorization is essential in the management of the cases. In most of the fetuses, cyst dimensions are small and they regress spontaneously.

Nevertheless, prognosis and therapy of these lesions are quite variable. Some rare complications such as ovarian torsion may require surgical intervention. ${ }^{4,5}$ Cystic abdominal masses are easily evaluated radiologically by ultrasonography, CT scan and MRI. Ultrasound is a very sensitive and specific radiological imaging modality used not only for the diagnosis but also for the follow-up of these cysts, even in the prenatal period. Ultrasonography is a sensitive and specific radiological assessment that provide several radiological features of the lesion. Once an abdominal mass is suspected, ultrasonography should be performed for an initial radiological evaluation. It is feasible and reveals fluid-filled cystic lesion. Computed Tomography and Magnetic Resonance Imaging could be helpful and essential in order to obtain a better features description of the mass. Additional information including lesion origin, its relationships, and its adhesion to visceral organs, this can be useful if laparoscopic surgery is considered. ${ }^{6,7}$ In this case, the investigation began with a simple abdominal X-ray that found a ground glass appearance in the abdominal cavity, as a sign of ascites. The investigation performed with USG, it found a fluid collection with internal echo all over the abdominal cavity with septas and cyst, suspect a mesenteric cyst.
The choice of treatment is total excision of the cyst whenever feasible because of the risk of recurrences. In fact, the sole aspiration of the cyst is not indicated and complete removal of the cyst is essential and is reported as a procedure of choice to avoid the cyst recurrence as well as its malignant transformation. The goal of surgical therapy for mesenteric cysts is complete excision of the mass. The preferred treatment of mesenteric cysts is enucleation, even though intestinal resection is frequently required to ensure that the remaining bowel is viable. Bowel resection may be required in $50-60 \%$ of children with mesenteric cysts, whereas resection is necessary for about one-third of adults. Any resulting mesenteric defect must be closed to prevent an internal hernia. If enucleation or resection is not possible because of the size of the cyst or because of its location deep within the root of the mesentery, the third option is partial excision with marsupialization of the remaining cyst into the abdominal cavity. Approximately $10 \%$ of patients require this form of therapy. If marsupialization is performed, the cyst lining should be sclerosed with $10 \%$ glucose solution, electrocautery, or tincture of iodine to minimize recurrence. Partial excision alone with or without drainage is not indicated because of the high recurrence rate associated with these procedures. ${ }^{1,8,9}$

Laparoscopic management of mesenteric cysts is also being reported. If necessary, depending on the expertise in laparoscopic surgery in children, laparoscopy could be used to localize the cysts, and resection could be performed through a small laparotomy or via an extended umbilical incision. Furthermore, reports suggest successful drainage and marsupialization of mesenteric cysts, which avoids enterotomy. Management of mesenteric cysts by ultrasound-guided drainage has also reported being successful. In this case, he underwent laparoscopic diagnostic and drainage ascites. After drainage ascites continued with laparotomy exploration. A transverse incision at supra umbilical and abdominal exploration was conducted. A Patient underwent exploratory laparotomy, which revealed a big lobulated mesenteric cyst in the mesentery of sigmoid, extending in the mesentery of descending colon and send the specimen to pathology anatomy for diagnostic. Day 4 after surgery, the result of pathology anatomy showed histomorphology confirmed a mesenteric cyst from the sigmoid colon..$^{9,10}$

The outcome of the mesenteric cyst except for malignant cystic mesothelioma, all mesenteric cysts are benign and their total excision is usually curative. However, benign cystic mesotheliomas and lymphangiomas have a high tendency to recur 
if they are incompletely resected. Hebra et al., who followed 13 patients with mesenteric lymphangiomas and 6 with omental lymphangiomas from 1 month to 17 years, reported $3(16 \%)$ recurrent mesenteric lymphangiomas. Similarly, Ross et al. observed a recurrence in 12 of 25 patients (48\%) reported in the literature after resection of benign cystic mesotheliomas. Although in the long term, no metastatic potential has ever been demonstrated with lymphangiomas or benign cystic mesotheliomas, a few patients have died from the progressive local enlargement. In the literature, the malignant transformation of mesenteric cysts has been suspected in 4 cases. In one of them only was a malignant transformation from a previous benign enteric cyst truly objectivate. In the remaining 3, 1 was arising from a Müllerian duct remnant, and 2 were of unknown origin. ${ }^{1,2,10}$ After surgery, the patient was admitted in the Pediatric Intensive Care Unit (PICU) in this case. The enteral feeding was postponed until 2 days. On the second days after surgery, he was in good condition, no fever, no bloating, and the bowel sound quite good. He tried to drink and well tolerated. He was admitted to the ward. On the $7^{\text {th }}$ day's post-surgery, he was discharged from hospital in good condition. Blood culture revealed no growth, and the pathology anatomy result revealed the mesenteric cyst, and no malignancy cell was founded. The parent worried about the outcome of the disease.

\section{SUMMARY}

A 2-year-old boy was hospitalized with a history of the bloated abdomen and dull ach pain on the left side of the abdomen since I year before admitted. Physical examination revealed distended of abdomen and ascites. Laboratory investigation showed normal limit. Ultrasonography investigation showed cystic lesion and fluid collection in the cavum abdomen. It was confirmed with a CT scan. We diagnosed the patient with ascites permagna et causa suspected mesenteric cyst. After the diagnosis, a laparoscopic exploration and drainage ascites and total resection of the cyst were performed. Seven days after surgery, the patient was in good condition and discharged from the hospital. No distention of the abdomen observed.

\section{CONFLICT OF INTEREST}

The authors declare that there is no competing interest regarding the manuscript

\section{FUNDING}

The authors are responsible for the funding of study without the involvement of grant, scholarship, or any other resource of funding.

\section{AUTHOR CONTRIBUTION}

All of authors are equally contributed to the study from the conceptual framework, data gathering, surgery, reporting the outcome, as well as manuscript preparation.

\section{DAFTAR PUSTAKA}

1. de Perrot M, Brundler M, Totsch M, Mentha G, Morel P. Mesenteric Cyst, Toward Less Confusion?. Dig Surg. 2000;17(4):323-328

2. Pithawa AK, Bansal AS, Kochar SPS. Mesenteric Cyst: A rare intra-abdominal tumor. Elsevier. 2014;70(1):79-82

3. Light DE, Pianki FR, Ey EH. Abdominal mass in an infant. J Am Osteopath Coll Radio 2015;4(1):23-25.

4. Ghritlaharey RK, More SK. Chylolymphatic cyst of mesentery of terminal ileum: a case report in 8 year-old boy. J Clin Diagn Res. 2014;8(1):ND05-7

5. Magno-Junior C, Batista CA, Leite GF, Lima HA, Paula JF, Kim MP, et al. [Mesenteric cyst: case report and review of the literature]. Arq Bras Cir Dig. 2012;25(2):137-8.

6. Prakash A, Agrawal A, Gupta RK, Sanghvi B, Parelkar S. Early management of mesenteric cyst prevents catastrophes: a single centre analysis of 17 cases. Afr J Paediatr Surg. 2010;7(3):140-3

7. Pampal A, Yagmurlu A. Successful laparoscopic removal of mesenteric and omental cysts in toddlers: 3 cases with a literature review. J Pediatr Surg 2012;47(8):e5-8.

8. Liaqat N, Latif T, Khan FA, Iqbal A, Nayyar SI, Dar SH. Enteric duplication in children: a case series. Afr J Paediatr Surg 2014;11(3):211-4

9. Mohanty SK, Bal RK, Maudar KK. Mesenteric cyst: an unusual presentation. J Pediatr Surg. May 1998;33(5):792-793

10. Raghupati RK, Krishnamurthy P, Rajamani J, et al. Intraabdominal cystic swelling in children-laparoscopic approach, our experience. J Indian Paediatr Surg. 2003;8:213-217

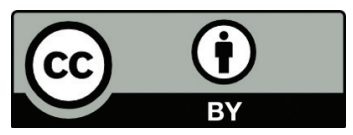

This work is licensed under a Creative Commons Attribution 